\title{
Solar Energy-Assisted Electrochemical Splitting of Water
}

\author{
Some Energetical, Kinetical and Catalytical Considerations Verified \\ on $\mathrm{MoS}_{2}$ Layer Crystal Surfaces \\ H. Tributsch \\ Fritz-Haber-Institut der Max-Planck-Gesellschaft Berlin, Germany \\ (Z. Naturforsch. 32 a, 972-985 [1977] ; received July 9, 1977)
}

\begin{abstract}
Kinetical, energetical and solid state considerations were elaborated in a search for suitable electrodes for the oxidation of water with visible light. They led to layer type transition metal dichalcogenides and to the photo-electrochemical utilization of optical d-d-transition which do not break chemical bonds. $\mathrm{MoS}_{2}$ was selected as a promising compound and it was actually shown to react with water on illumination with light between 400 and $715 \mathrm{~nm}$. At low electrode potentials the liberation of small quantities of molecular oxygen was traced with polarographic techniques. The main portion of the oxidation products of water was, however, found to be lost for the oxydation of crystal bound sulfur to sulfate. An improvement of this situation in favour of an increased rate of oxygen evolution has been accomplished by means of a redox-catalyst, tris (2,2'-bipyridine) ruthenium (II), which is oxidized through photochemically generated holes from the $\mathrm{MoS}_{2} 4 \mathrm{~d}_{\mathrm{z}^{2}}$ valence band and channels them into oxygen-evolution through nucleo-philic addition of hydroxide. A molecular mechanism was elaborated for the photoelectrochemical oxidation of water on $\mathrm{MoS}_{2}$ and its conclusions tested by investigating the oxidative behaviour of various additional layer-type transition metal compounds. It involves a hole mediated valence change of molybdenum with an intermediate formation of a 5-valent molybdenum hydroxide. A basic kinetical and energetical similarity is suggested with the function of manganese reaction centers in photosynthesis.
\end{abstract}

\section{Introduction}

The aim of a photochemical or photoelectrochemical splitting of water for the purpose of generating a storable fuel has recently attracted considerable scientific interest. The mechanism of photosynthesis in plants and some bacteria still remains the only existing efficient mechanism of this type, but some progress has been achieved with several interesting model systems: Most work has been concentrated on the photoelectrochemical splitting of water on illuminated surfaces of oxides such as $\mathrm{TiO}_{2}{ }^{1-9}, \quad \mathrm{SnO}_{2}{ }^{10}, \quad \mathrm{SrTiO}_{3}{ }^{11,12}$ and $\mathrm{KTaO}_{3}{ }^{13}$. The main limitation of these systems is that they can only be operated with UV-light. If a recently published experiment can be confirmed, $\mathrm{GaP}$ covered with a gold film ${ }^{14}$ seems to overcome this spectral problem, it suffers, however, from the problem of corrosive selfdestruction. Organic compounds have also been suggested as possible catalysts for the photochemical splitting of water. Calvin's group has experimented with some success with a synthetic manganese containing compound ${ }^{15}$ and Creutz and Sutin ${ }^{16}$ suggest the tris (bipyridine) ruthenium III complex which liberates oxygen from

Reprint requests to Dr. H. Tributsch, Fritz-Haber-Institut, Faradayweg 4-6, D-1000 Berlin 33 . hydroxide ions ${ }^{16}$. The complex may be generated photochemically with light below $560 \mathrm{~nm}$ and subsequent oxidation. Since the reduced compound would potentially be capable of reducing protons to hydrogen the authors suggest to capture the electron in the conduction band of a semiconductor and to reduce protons at a $\mathrm{Pt}$-counterelectrode. A problem which these interesting systems will face, even if high quantum-efficiencies could be accomplished, will be the high costs to replace the valuable organic compounds lost in inevitable photochemical side reactions. Up to now the development of model systems for solar energy mediated or -assisted oxidation of water was mostly experimentally and not theoretically oriented. Attemps to pinpoint substrates for the photochemical oxidation of water through theoretical reasoning, that is through theoretically elaborating and experimentally verifying molecular concepts on the molecular mechanism of water splitting, could be a valuable tool to improve the knowledge in this field, and a first try is presented in this publication.

Some among the large number of considerations which served to identify a suitable substrate are certainly oversimplified and may even be incorrect. The fact that the attempt was partially successful and that additional substrates may be identified 


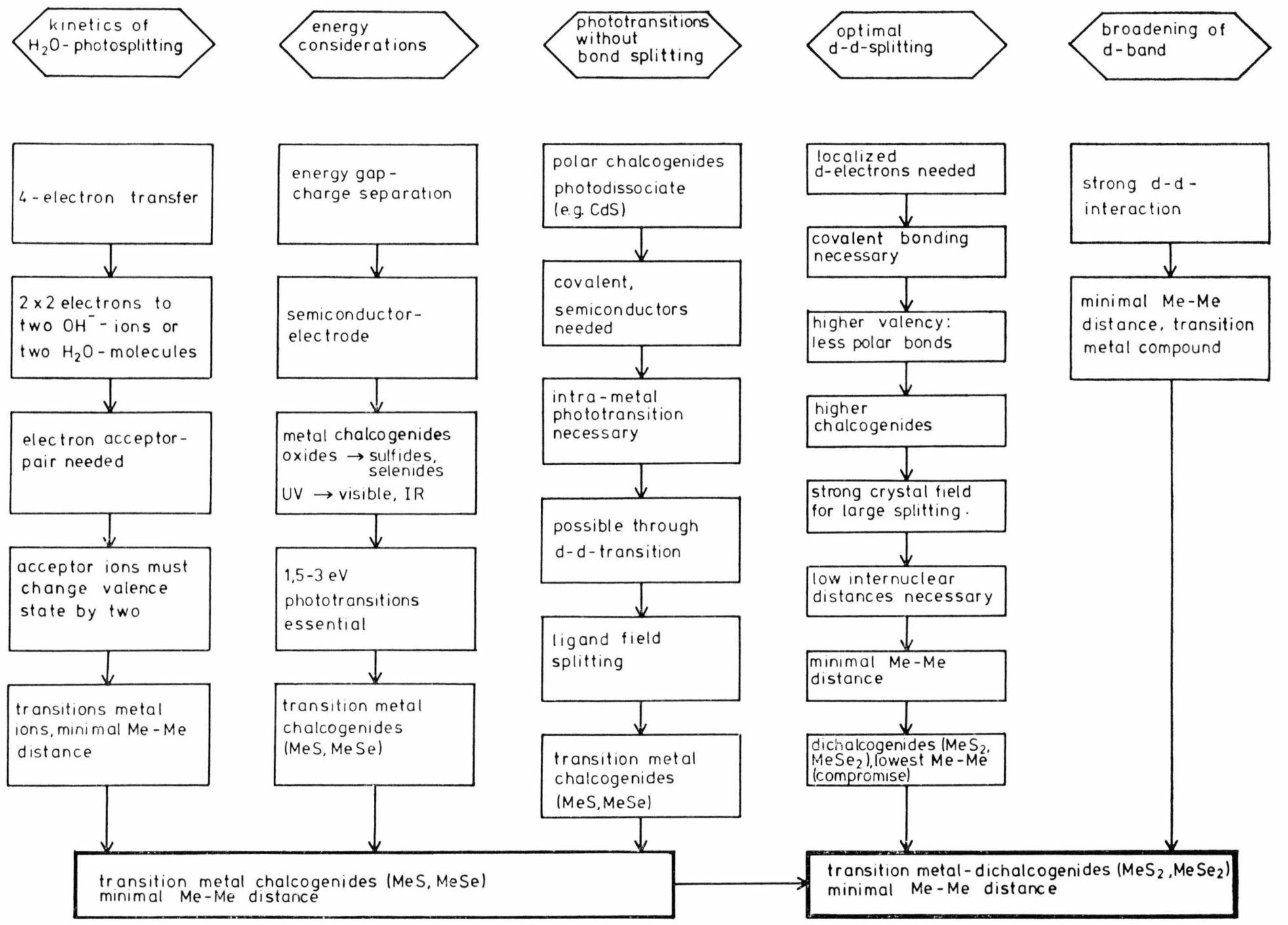

Fig. 1. Simplified scheme, visualizing the lines of considerations, arranged in 5 columns, leading to the criterion which was applied for the identification of a target substrate for a photoelectrochemical oxidation of water. 
with the applied procedure, however, justify a short discussion of the applied criteria.

\section{Photooxidation of Water: Theoretical Considerations}

According to the authors knowledge a systematical, theoretical attempt to define the necessary properties of a katalyst for the photosplitting of water has not yet been made. Its characteristic behaviour is bound to include energetically and kinetically suitable electron-transfer properties from water to photochemically or electrically emptied electron orbitals, suitable light absorption properties and the capacity to separate excited electrons from reactive oxidation products. Additional and essential conditions would be that the photocatalyst should be chemically stable, easy to synthesize and cheap to produce. The main considerations which have been employed during the tentative encirclement of a suitable catalyst are arranged in Figure 1. They are grouped in five columns according to the problem investigated. The first column analizes the kinetical and sterical preconditions needed for a transfer of four electrons during oxidation of water. The second defines optical and energetical conditions which have to be satisfied by a photocatalyst during photoabsorption and for charge separation. In the third column a search is made for a suitable phototransition which can be produced with visible light, but does not break chemical bonds. In the fourth the conditions are analized which lower the energy level of the groundstate to the oxidation potential of water. In the fifth it is investigated how these levels can be broadened into an energy band for better electrochemical reactivity and photoconduction.

The conditions and limitations imposed by these considerations were then summarized to derive a theoretical criterion, which was then applied for the identification of a possible photocatalyst. In the following the line of considerations will shortly be sketched:

In earlier work on energy conversion in photosynthesis ${ }^{17}$ the author has expressed the opinion that the possibility of separating excited electrons from reactive holes should be an essential prerequisite for an efficient quantum conversion. It appeared therefore reasonable to search for a suitable photoreaction in the interface of a crystallized material and not in a homogeneous solution containing photoreactive compounds. Only semiconduc- tors combine energetically suitable light-induced electronic transitions with conductivity properties. Among these, only compounds of transition elements and electronegative elements such as oxygen, sulphur, selenium and tellurium seem to be potentially suitable as photocatalysts for the oxidation of water. In these, the energetical positions of the valence band and the conduction band can be varied in a wide range according to composition and crystal structure.

A huge amount of compound semiconductors in consideration, on which little or no experimental information exists had to be screened theoretically: It is well known that the forbidden band gap of semiconductors tends to decrease gradually on transition from oxygen to sulphur, selenium and tellurium as crystal components. Oxygen compounds usually absorb in the ultraviolet and blue region (e.g. $\mathrm{TiO}_{2}, \mathrm{ZnO}, \mathrm{SnO}_{2}$ ) and were, therefore, excluded. The important condition that electronic transitions from the valence band to the conduction band occur in the visible region is best satisfied by sulphur compounds (e.g. $\mathrm{CdS}, \mathrm{Cu}_{2} \mathrm{~S}, \mathrm{PbS}$ ). Selenium and tellurium compounds also adsorb visible light, but their energy gaps decrease and the edge of their valence band shifts towards less positive redox-potentials. They are consequently less probable but still possible candidates for photocatalysis of oxygen evolution. These energetical considerations (second column in Fig. 1) therefore suggest a search for a suitable photocatalyst among transition metal chalcogenides, especially sulfides.

The peculiarity of the 4-electron-oxidation mechanism of water to molecular oxygen makes it reasonable to consider the following additional limitation with respect to the properties of the photocatalyst (first column in Fig. 1): Since two times two electrons have to be transferred from two neighbouring $\mathrm{OH}^{-}$ions or $\mathrm{H}_{2} \mathrm{O}$ molecules for an energetically favourable liberation of oxygen molecules it is - in agreement with Calvin's model for the mechanism of the manganese-complex in photosynthesis (15) - reasonable to request the existence of pairs of acceptor ions which can change their valence state by two.

Such acceptor ions have to be searched for among transition metals and their distance should be as small as possible because of the small $\mathrm{O}-\mathrm{O}$ distance in oxygen molecules. (Conclusion of column 1 in Figure 1.) 


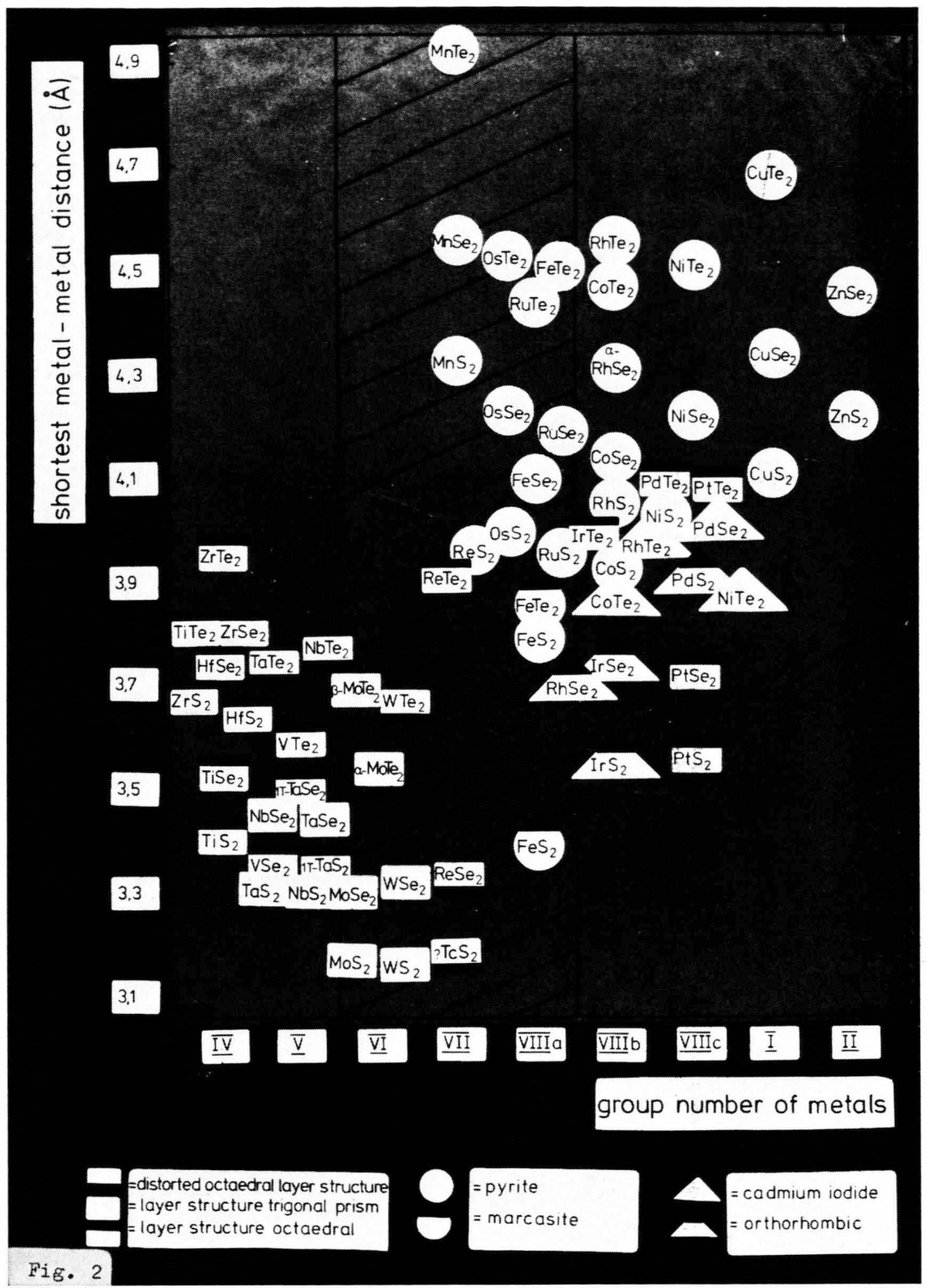

Fig. 2. Shortest metal-metal distances of approximately 70 transition metal dichalcogenides arranged according to the group numbers of metals. Those compounds lying within the dashed area (valence change from 4 to 6 possible) and having the shortest metal-metal distance are identified as the most promising catalysts.

In order to get phototransitions without splitting chemical bonds (column 3 ) it would be necessary to abandon polar semiconductors (such as CdS) completely, to turn to covalent semiconductors and to search for suitable intra metal $\mathrm{d}-\mathrm{d}$ excitations. Only transition metal compounds could provide them.

Conditions outlined in columns $1-3$ of Fig. 1 are consequently limiting suitable substrates for photocatalysis to transition metal chalcogenides with a minimal metal-metal distance. The addi- tional conditions that the splitting between d-orbitals should be sufficiently wide for optical transitions (column 4) and the energy band derived from dorbitals sufficiently wide for electrical conduction (column 5) is putting emphasis on the requirement that there should be a strong metal-metal interaction and consequently a small metal-metal distance in the substrate. Since there is a larger internuclear distance and less bonding interaction in polar than in covalent crystalline material it would also seem reasonable to concentrate the search on 
the more covalent divalent transition metal chalcogenides than on less covalent monovalent ones.

The entire scheme of considerations of Fig. 1 suggests consequently a search for suitable photocatalysts among those transition metal dichalcogenides with a minimal metal-metal distance.

\section{The Identification of a Target-Substrate}

The metal-metal-distances of approximately 70 dichalcogenides have been collected from the literature ${ }^{18-20}$, plotted according to the group number of metals and classified according to the crystal structure (Figure 2). It can be seen from this presentation that pyrite type crystal structures with considerable polar contribution to bonding do not meet the condition elaborated. The minimal metal - metal distance is found in layer type dichalcogenides of covalent bonding character, the most favourable compounds being $\mathrm{WS}_{2}, \mathrm{MoS}_{2}$ and $\mathrm{TcS}_{2}$. They also satisfy the condition that the valence state of transition metals can be changed by two (dashed area in Figure 2).

None of these or related layer type compounds has up to now been investigated with respect to electrochemical or photochemical properties.

$\mathrm{TcS}_{2}$ was eliminated because of its scarcity. No larger crystals, desirable for photoelectrochemical experiments were obtainable of $\mathrm{WS}_{2}$. Our interest was consequently concentrated on $\mathrm{MoS}_{2}$ which is widely used as a solid state lubricant like graphit and is available in the form of large natural layer crystals.

The basic structural unit of $\mathrm{MoS}_{2}$ is a sandwich of three planes: sulfur-molybdenum-sulphur. The $\mathrm{S}-\mathrm{Mo}-\mathrm{S}$ sandwiches are only loosely bound together through van der Waals-forces. A $\mathrm{MoS}_{2}$ surface, produced by cleaving the layer crystal is consequently formed by a monolayer of sulphur, the atoms of which are firmly bound to six sulphur neighbours in the same plane at $3.16 \AA$ and to three Mo neighbours at 2.42 in the middle of the $\mathrm{S}-\mathrm{Mo}-\mathrm{S}$ sandwich ${ }^{18}$. There are no free dangling orbitals on the surface for the formation of additional chemical bonds. The surface of the layer crystal $(\perp c)$ may therefore be called a van der Waals-surface. MoS $\mathrm{S}_{2}$ occurs in two modifications. The hexagonal $2 \mathrm{H}$-polytype has two sandwiches per unit cell along the c-axis, the rhombohedral 3Rpolytpe three sandwiches.
The energy band structure of the selected $\mathrm{MoS}_{2}$ compound ${ }^{21-23}$ appeared to meet the conditions introduced into the concept which was applied for its identification (Figure 3). The conduction-band, approximately $3 \mathrm{eV}$ wide, is based on four of the six Mo $4 \mathrm{~d}$ orbitals $\left(4 \mathrm{~d}_{x y} / 4 \mathrm{~d}_{x^{2}-y^{2}}\right)$, with anti bonding admixtures of $\mathrm{S} 3 \mathrm{p}$ orbitals. Somewhat less than $2 \mathrm{eV}$ below (our experiments yielded a gap for optical transitions of $1.75 \mathrm{eV}$ ) there is the non bonding Mo $4 \mathrm{~d}_{z^{2}}$ orbital holding two electrons per $\mathrm{MoS}_{2}$. It is approximately $1 \mathrm{eV}$ wide. Below and slightly overlapping $(0.1 \mathrm{eV})$ with the Mo $4 \mathrm{~d}_{z^{2}}{ }^{2-}$ band is the principal S $3 p$ valence band. The interesting fact that $\mathrm{d}-\mathrm{d}$ transitions form the direct absorption edge is well supported by recent band calculations $21,24,25$ as well as by paramagnetic resonance measurement of holes ${ }^{26}$. The absorption of visible light creates consequently holes in the valence band and electrons in the conduction band without affecting essential chemical bonds in the sulfide surface.

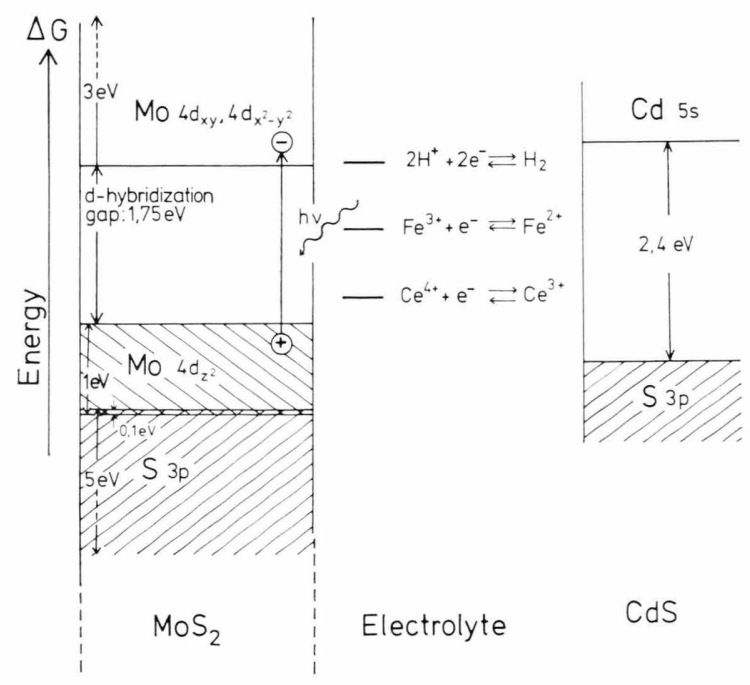

Fig. 3. Simplified energy band scheme of $\mathrm{MoS}_{2}$ as compared with CdS and redox-levels in the electrolyte.

\section{Experimental}

The experiments were performed with naturally grown $\mathrm{MoS}_{2}$ crystals which were isolated from samples obtained from Mangualde, Portugal and Kingsgate, Australia, and with synthetic specimen of $\mathrm{MoSe}_{2}, \mathrm{ZrSe}_{2}$ and $\mathrm{HfSe}_{2}$ (obtained from the Institute of Crystallography (Prof. R. Nitzsche), University of Freiburg, produced by Iodine trans- 
port from the gas phase. Selected layer crystals of surface areas between 0.5 and $1 \mathrm{~cm}^{2}$ were provided with an electrical contact and embedded into a teflon mounting. The procedure is described in ${ }^{27}$. The crystal surface, exposed to the electrolyte, was the surface which is produced through natural splitting $(\perp c)$, it was renewed either by splitting the crystal (with the help of a tape which is subsequently removed) or by means of an abrasive technique, through a water jet, which is directed towards the surface. For the qualitative and quantitative determination of photoelectrochemically liberated oxygen the polarographic technique was used. To warrant a sensible measurement, the dropping mercury electrode was incorporated into the electrochemical cell which was isolated against access of oxygen from the atmosphere. The illuminated $\mathrm{MoS}_{2}$ and the dropping mercury electrode were then used alternatively.

For the optical studies a $150 \mathrm{~W}$ Xenon lamp and a high intensity Bausch and Lomb monochromator were used. Electrochemical experiments were performed potentiostatically and with a standard set up for dynamical current-voltage measurements.

\section{Photoelectrochemical Reaction of Water on the $\mathrm{MoS}_{2}$ Surface}

A good photo-effect could be established for $\mathrm{MoS}_{2}$ electrodes in the visible spectral region between 400 and $715 \mathrm{~nm}$. It was cathodic for p-type samples (dashed curves in Fig. 4) and anodic for n-type samples ${ }^{27}$. At 2 Volts anodical potential quantum efficiencies of $60 \%$ could be measured for the generation of the photocurrent in spite of the loss of 20 to $30 \%$ of light through reflection from the metallic bright surface. In contrast to polar metal sulfides such as CdS, molecular sulphur was - apart from traces - not the product of anodical photoelectrochemical dissolution. Since the electrolyte remained clear and the sulfide surface metallic bright even after passage of more than 100 Coulombs of photocharge ${ }^{27}$ a photoelectrochemical reaction of water on the $\mathrm{MoS}_{2}$-surface was left as the only possible mechanism that could have produced a transfer of electrical charge across the electrodeelectrolyte interface. Two different reactions had to be taken into consideration, one leading to the photoelectrochemical formation of sulfate, the other to the photoelectrochemical liberation of molecular oxygen from water:

$$
\begin{aligned}
& \mathrm{MoS}_{2}+8 \mathrm{H}_{2} \mathrm{O}+18 h^{+} \stackrel{18 h v}{\longrightarrow} \mathrm{Mo}(\mathrm{VI})+2 \mathrm{SO}_{4}{ }^{2-} \\
& \mathrm{MoS}_{2}+2 \mathrm{H}_{2} \mathrm{O}+4 h^{+} \stackrel{4 h v}{\rightarrow} \mathrm{MoS}_{2}+\mathrm{O}_{2}+4 \mathrm{H}^{+} .
\end{aligned}
$$$$
+16 \mathrm{H}^{+}
$$

Reaction (1) leading to the formation of sulfate turned out to be the predominant reaction in longtime experiments performed at an anodic electrode potential between 0.3 and 0.5 Volt and operating of up to 19 days ${ }^{27}$.

Reaction (2) could, as the following experimental results will show, also be detected although the liberation of oxygen was found to be limited to the region of the anodical onset of the photocurrent $(<0.5 \mathrm{Volt})$ where the quantum efficiency for the photoelectrochemical reaction is low (Figure 4). On application of a higher voltage to the illuminated $\mathrm{MoS}_{2}$ electrode there was even a decrease of oxygen concentration in the electrolyte, apparently due to its reaction with intermediates of the sulphuroxidation.

The determination of oxygen liberation during illumination with visible light $(\lambda>420 \mathrm{~nm})$ was made with the polarographic setup, incorporated into the electrochemical cell. The experiments were performed in such a way that the $\mathrm{MoS}_{2}$ electrode was alternatively kept in the dark or illuminated

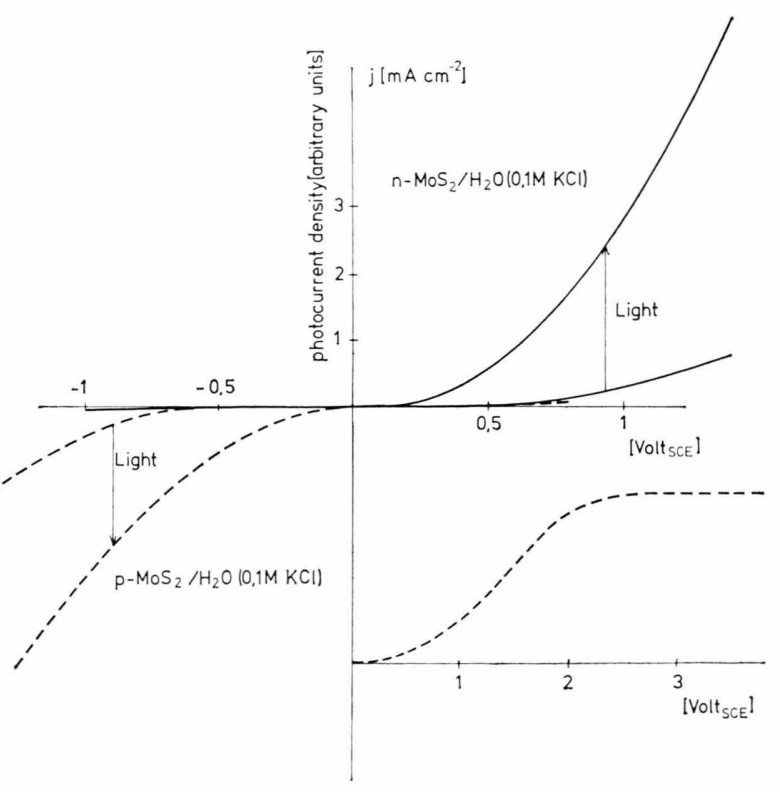

Fig. 4. Current-voltage characteristics for p-type (dashed curves) and n-type $\mathrm{MoS}_{2}$-electrodes with and without illumination. Inserted with condensed voltage scale is the limiting photocurrent behaviour of $\mathrm{n}-\mathrm{MoS}_{2}$. 
Table 1. Rate of current increase of the first polarographic oxygen reduction step during illumination of $n$-MoS $\mathrm{S}_{2}$ with visible light $(\lambda>415 \mathrm{~nm})$.

\begin{tabular}{|c|c|c|c|c|c|c|c|}
\hline \multirow{2}{*}{$\begin{array}{l}\text { electrode } \\
\text { number }\end{array}$} & \multirow{2}{*}{$\begin{array}{l}\text { electrode } \\
\text { potential } \\
\text { vS SCE [V] }\end{array}$} & \multicolumn{4}{|c|}{$\mathrm{di} / \mathrm{dt}\left[10^{-7} \mathrm{~A} / \mathrm{h}\right]$} & \multirow{2}{*}{$\begin{array}{l}\text { initial } \\
\mathrm{pH} \text { of } \\
\text { electrolyte }\end{array}$} & \multirow{2}{*}{ electrolyte } \\
\hline & & dark & light & dark & light & & \\
\hline 1 & 0,3 & 0,39 & 4.38 & 0,1 & 4,35 & 7 & \multirow{5}{*}{$0,1 \mathrm{M} \mathrm{KCl}$} \\
\hline 2 & 0,3 & 0,52 & 4,58 & 0,9 & 3,55 & 7 & \\
\hline 3 & 0,3 & 0,4 & 4,0 & 1,2 & 5,2 & 7 & \\
\hline 4 & 0,4 & 0,59 & 3,5 & 0,85 & 3,39 & 7 & \\
\hline 5 & 0,3 & 0,91 & 3,2 & 0,78 & 3,0 & 10 & \\
\hline 6 & 0,4 & 0.5 & 4,4 & 0,64 & 2,1 & 9 & $\begin{array}{l}\text { borate buffer } \\
0,1 \mathrm{M} \mathrm{KCl}\end{array}$ \\
\hline 7 & 0.4 & 0,79 & 8,4 & 0,4 & 4,0 & 9 & \multirow{2}{*}{$\begin{array}{l}\text { bor ate buffer } \\
0,1 \mathrm{M} \mathrm{KCl} \\
+2,6 \cdot 10^{-2} \mathrm{M} \mathrm{Ru}(\text { bipy) } \\
3\end{array}$} \\
\hline 8 & 0,3 & 1,2 & 8,0 & 1,6 & 8.8 & 9 & \\
\hline
\end{tabular}

for time periods of one hour and the polarographic oxygen curve in the electrolyte taken without any interference in the setup of the system.

The results of eight independent experiments, performed with different n-type crystals are represented in Table 1 in which the increase of the oxygen reduction current (first polarographic step) is plotted for subsequent periods of dark and light: Some ordinary diffusion of atmospheric oxygen into the cell persisted instead of careful isolation attempts by way of the dropping mercury and the hammer activated capillary system. The rate of photogenerated oxygen is, however, an order of magnitude higher and can easily be distinguished (Figure 5).

Attempts to measure the quantum efficiency of photoassisted generation of oxygen from water quantitatively met with some difficulties, since there were always a gaseous phase and gas-bubbles in equilibrium with the electrolyte solution, the total volume of which was difficult to estimate. The efficiency of photoelectrical generation of oxygen was found to be of the order of magnitude to be expected from the amount of photocurrent passed. At anodical electrode potentials between 0.3 and 0.4 Volts, measured against the saturated calomel

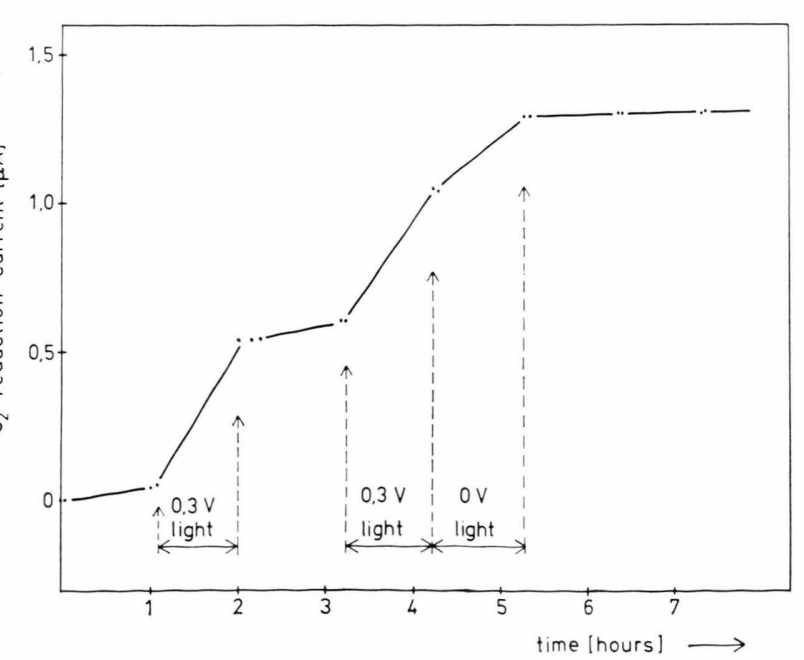

Fig. 5. Increase of oxygen reduction current (first step of polarographic wave) during illumination of $\mathrm{n}-\mathrm{MoS}_{2}$ with visible light. 
electrode, and with a neutral electrolyte this efficiency amounted to one per cent or less. The remaining portion of photogenerated electrons and holes is not converted into a photocurrent in this range of the current-voltage characteristics and apparently lost through recombination. However, several experiments indicated a photoelectrochemical oxygen production which exceeded the value expected from the simple Faraday law. One possible explanation would be a simultaneous production of divalent molybdenum ions. A clarification of this unresolved aspect of photoassisted oxidation of water will be attempted with an improved method for quantitative oxygen determination.

The rate of light assisted oxygen liberation gradually decreased in the course of several subsequent one-hour-periods of illumination. This might be due to a progressive shift of the reaction mechanism from the ideal van der Waals surface to half crystalline sites in which the weaker bonding situations should facilitate formation of sulfate.

\section{Representative Experiments with Additional Layer-Type Transition Metal Dichalcogenides}

In order to test the validity of the derived concept of a substrate's properties required for photoelectrochemical oxidation of water and in order to pinpoint the detailed reaction mechanism with water, several additional layer-type transition metal dichalcogenides were investigated.

To get some insight into the energetical requirements for a mechanism of light induced water oxidation through holes from a d-band we were interested to see how a system would behave which provides a d-band at less positive redox potentials than $\mathrm{MoS}_{2}$. The scheme of Fig. 2 identifies $\mathrm{MoSe}_{2}$ as suitable candidate. Compared with $\mathrm{MoS}_{2}$ (Fig. 3) the low Mo $-\mathrm{d}_{z^{2}}$ band is shifted upwards in this compound and the $\mathrm{d}-\mathrm{d}-$ gap is decreased to approximately $1.5 \mathrm{eV}$.

Nevertheless, as experiments have shown, even with infrared illumination between 750 and $900 \mathrm{~nm}$ the efficient anodic photoelectrochemical reaction involves the oxidation of water leading to a formation of selenic acid ${ }^{31}$. In contrast, the polar compound CdSe which provides photogenerated holes in the selenium s-band does not show any reactivity with water but is anodically liberating selenium in molecular form.
Characteristical current-voltage dependences of semiconductor electrodes are essentially determined by the variation of the space charge layer and not by a change of the surface Helmholtz layer which would produce a relative shift of energy levels in the semiconductor-electrolyte interface. The relative energetic position of $\mathrm{d}$-bands and the electronic levels of water do therefore not markedly change with the applied electrode potential. It can consequently be concluded that in contrast to holes from $\mathrm{s}$ bands which apparently only react with water if their energy position is considerably (of the order of $1 \mathrm{eV}$ in $\mathrm{TiO}_{2}, \mathrm{ZnO}, \mathrm{SnO}_{2}$ ) more positive than the thermodynamical oxidation potential of water, holes from a d-band of $\mathrm{MoS}_{2}$ and $\mathrm{MoSe}_{2}$ efficiently react with water as if it were a simple reducing agent like hydroquinone.

Holes in the d-valence band of $\mathrm{MoS}_{2}$ are not available at more positive redox potentials than that in the valence band of CdS (Figure 3). From an energetical point of view there is consequently no reason why holes from $\mathrm{MoS}_{2}$ should react efficiently with water and holes from CdS and similar polar sulfur compounds not. The oxidation of water cannot easily be explained as a two-electron reaction for which the thermodynamic redox potential of $1.23 \mathrm{eV}$ is calculated. There should be two distinguishable and successive electron transfer steps. A one-electron oxidation of an $\mathrm{OH}$-ion is, on the other hand, energetically impossible without a simultaneous formation of a chemical bond between the hydroxyde and the electrode surface which would account for the energy difference. Bond formation depends on the availability of bonding orbitals. Since there are no dangling orbitals on $\mathrm{MoS}_{2}$-van der Waals surfaces $(\perp c)$, which form the interface predominantly exposed to water, one should expect layer crystals to be even less favourable reaction partners than polar sulfides which provide a lot of free surface orbitals and where, in addition, existing crystal bonds are broken in the presence of holes. The only answer to these apparent contradictions is that hole reactions from d-bands must be favoured by some kinetical factors which are completely missing in the case of hole reactions from sbands of polar semiconductors.

Two details have been elaborated which could play a crucial role in this mechanism: A closer examination of the sterical situation for electron transfer and of electron density distributions on 
layer sulfide surfaces indicates that sulfur atoms may play a special role as bridges for electron transfer between water on the crystal surface and the d-electron state of the transition metal which forms the second atom layer (Figure 9). A main argument for this conclusion is the recognition that there is not sufficient direct overlapping of orbitals between water and molybdenum d-states across the sulfur surface layer to warrant a highly efficient electron transfer. This consideration would, however, only apply if photoelectrochemical oxygen production would mainly occur on the van der Waals surface and not at crystal steps where the bonding situation is different. It is interesting to note that sulfur bridges also play an important role as bridges in biological electron transfer reactions.

The second observation was inspired by a theoretical consideration indicating that the four valent molybdenum in the surface $\mathrm{S}-\mathrm{Mo}-\mathrm{S}$ layer might become chemically instable as a consequence of the capture of a hole. Around a $\mathrm{MoS}_{2}$ unit cell there are only relatively few bonds which are linking it to the two dimensional crystal layers and towards the electrolyte there would be space for a confirmational expansion. It is therefore possible and probable that a capture of a hole which is equivalent to the transition of Mo(IV) to $\left.\mathrm{Mo}_{\mathrm{O}} \mathrm{V}\right)$ is accompanied by a redistribution of electron densities which would create an additional bond for the addition of an $\mathrm{OH}^{-}$ion.

In order to decide whether the sulfur bridge arrangement or a valency change of the transition metal is the critical factor for the photo-oxidation of water, experiments were initiated with group IV transition metal dichalcogenides (group V compounds have metallic character) (Figure 2).

If the charateristical layer structure with a sulfur, or selenium monolayer on the surface would be responsible for the reaction, sulfides or selenides of $\mathrm{Ti}$, Hf and $\mathrm{Zr}$ should also exhibit an anodical oxidation to sulfate and selenate. If, however, a valency change would be a necessary precondition for a hole reaction with water, group IV compounds should not be able to oxidize water, since they are placed outside the dashed area in Figure 2. Zirconium selenide $\left(\mathrm{ZrSe}_{2}\right)$ and Hafnium selenide $\left(\mathrm{HfSe}_{2}\right)$ were selected for the experiments because they had energy gaps comparable to that of $\mathrm{MoS}_{2}$ (1.7 and $2 \mathrm{eV}$ respectively). (The crystals available were synthetic specimen grown from the gas phase.)
Electrochemical measurements revealed a pronounced asymmetrical behaviour of both compounds. The exponentially rising anodical dark currents which already started at -0.1 to -0.2 Volts against the saturated calomel electrode indicated a largely p-type character for both compounds. Most interestingly, a red anodic selenium deposit rapidly forms. An anodical oxidation of water and the formation of selenic acid as it was observed with $\mathrm{MoSe}_{2}$ does consequently not occur as a principal reaction step. The layer type crystal structure and the special arrangement of sulfur atoms which form the surface monolayer and could function as bridges for electron transfer is consequently not the critical factor for the visible or infrared light induced photooxidation of water at $\mathrm{MoS}_{2}$ and $\mathrm{MoSe}_{2}$ surfaces. The clue for the photoreaction with water lies therefore in the valency change of Mo(IV) to $\mathrm{Mo}(\mathrm{V})$ which results as a consequence of a hole capture and induces a redistribution of chemical bonds.

Additional indications for conformational changes occuring on the surface of $\mathrm{MoS}_{2}$ and $\mathrm{MoSe}_{2}$-electrodes were actually found. Investigation of the direct currents of both compounds showed that it is occasionally superposed by trains of current pulses of exactly identical amplitudes but varying pulse lengths ${ }^{32}$. They are considerably larger than the electrode noise and their current density is of the order of one $\mu \mathrm{A} / \mathrm{cm}^{2}$. They appear with rectangular shape in the case of $\mathrm{MoS}_{2}$ electrodes and their risetime is faster than $\mu$ s. Their shape and behaviour pattern appears to be compatible with the assumption of bistable confirmational states in the electrode surface as it could be expected for Mo(IV) to Mo(V) transitions at critical concentrations of holes in macroscopical surface areas.

\section{Catalytical Improvement of Photoelectrochemical Oxygen Evolution}

The employed theoretical and experimental procedure has to be considered practicable, since it was actually possible to find a substrate which leads to the oxidation of water during illumination with visible light. The $\mathrm{MoS}_{2}$-system investigated turned out to be especially fovourable since low energy photons of only $1.75 \mathrm{eV}$ are sufficient to initiate the reaction. However, unfortunately, several disadvantageous secondary properties make the system 
behave deficiently in a way that, for the moment, it has to be considered far from practical significance and only of theoretical interest for mainly two reasons:

a) The anodical photocurrent characteristics which raises only very gradually with the applied potential makes it necessary to apply an external potential to the electrode for closed circuit operation. The electrical energy needed for the oxidation of water is still clearly lower than the thermodynamical dissociation energy $(E=$ $1.23 \mathrm{eV}$ ) by several tenths of an electron Volt. However, it amounts to an appreciable portion of the total energy.

b) The principal pathway of photoelectrochemical water oxidation leads to sulfate formation and not to oxygen liberation. The intermediates of water oxidation are apparently too reactive to leave the sulfur surface atoms of $\mathrm{MoS}_{2}$ untouched.

The first deficiency (a), which is due to an anodical flat-band potential of $\mathrm{MoS}_{2}$, and also indicates larger trapping losses in its space charge layer, could theoretically be reduced by changing the Fermi-level through appropriate doping of the material, by searching for crystallographically less disturbes electrode samples or - more favourable layer - type transition metal-dichalcogenides (Fig. 2 ), or by experimenting with the electrode propertiel through intercalation of the layer crystals.

The second deficiency (b) is a more serious one and became the centerpoint of a more detailed investigation: Since the high reactivity of oxidation products of water has to be considered the central problem, an oxidation of crystal-bound sulfur to

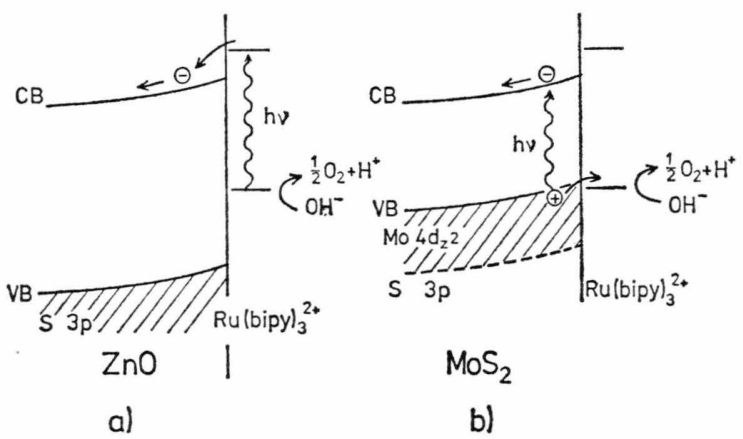

Fig. 6. Energy scheme for photoelectrochemical reaction in which Ru(bipy) ${ }_{3}{ }^{2+}$ acts as a sensitizer (a) and as a redox catalyst respectively (b). sulfate can only be suppressed in a reasonable way if the chemical steps leading to an oxidation of water to oxygen and protons could be made to proceed in a controlled way. Our interest was consequently concentrated on finding a suitable organic redoxcatalyst which could be photoelectrochemically oxidized on the surface of the layer crystal and would channel the energy of its positive charge into oxygen evolution from water through a well defined reaction. A favourable type of reaction appeared to be the hydroxide or water addition to $\mathrm{N}$-heterocycles ${ }^{28}$ and, as a test compound, the tris (bipyridine) ruthenium(II) complex was selected which has been investigated in some detail by Sutin and collaborators ${ }^{29,30}$. This compound, which absorbs light below $560 \mathrm{~nm}$, has attracted attention because in its oxidized state it is able to oxidize hydroxide ions to liberate molecular oxygen. Creutz and Sutin suggest to use this compound like a sensitizing dye and to capture its excited electron in the conduction band of a semiconductor with a large energy gap (Figure $6 \mathrm{a}$ ). In this way, absorption of visible light should lead to a liberation of oxygen. The way we intended to use this complex is different (Figure $6 \mathrm{~b}$ ). The visible light should be absorbed in the $\mathrm{MoS}_{2}$ electrode and holes in the low lying valence band, which are able to react with water, should, instead, be captured by Ru(bipy) ${ }_{3}{ }^{2+}$ ions, which - in their oxidized form - would oxidize water through a better controlled mechanism. A main difference between these two mechanism (Fig. $6 \mathrm{a}$ and b) would be that in the first case only a very small number of sensitizing $R u(\text { bipy })_{3}{ }^{2+}$ molecules will be able to participate in the energy conversion reaction, whereas in the second case holes which are generated in the Mo $4 \mathrm{~d}_{z^{2}}$-band of $\mathrm{MoS}_{2}$ should be transferable to the complex with high efficiency. This latter mechanism would not be a sensitization process, but a case of redox catalysis.

The oxidation of Ru(bipy) ${ }_{3}{ }^{2+}$ according to Fig. $6 \mathrm{~b}$ could actually be demonstrated. However, since both, the Ruthenium-complex with its redox potential of $1.20 \mathrm{~V}^{30}$ and water are photoelectrochemically oxidized at an anodically polarized $\mathrm{MoS}_{2}$ electrode, the oxidation of the complex did not produce an increase of the anodical photocurrents, when water was used as an electrolyte. To demonstrate the electrochemical oxidation of $\mathrm{Ru}$ (bipy) ${ }_{3}{ }^{2+}$ as a parallel reaction to the direct oxidation of water we turned to acetonitrile $\left(0.1 \mathrm{M} \mathrm{LiClO}_{4}\right)$ as an electro- 


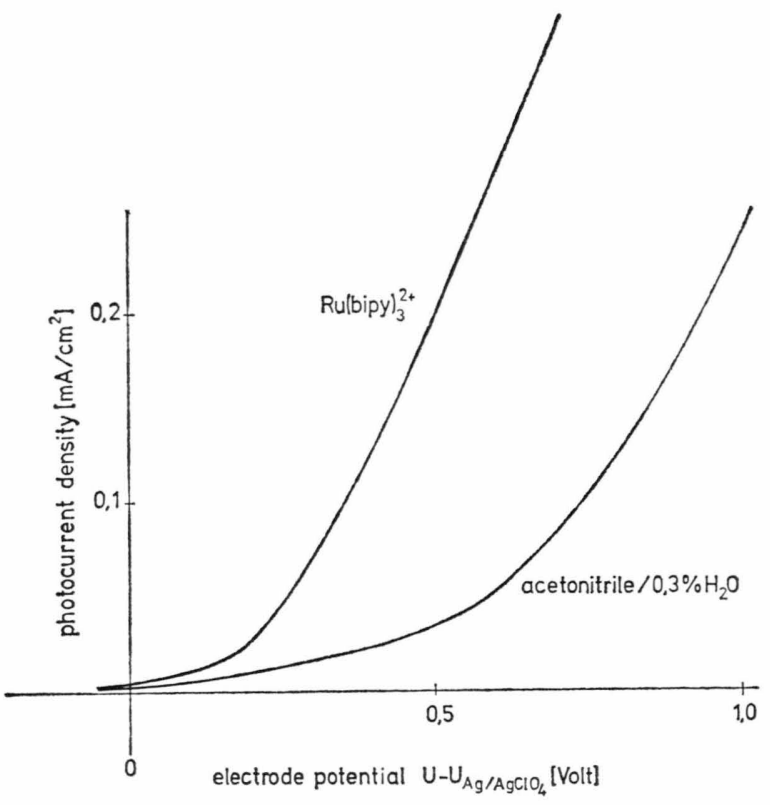

Fig. 7. Effect of Ru(bipy) ${ }_{3}{ }^{2+}$ on the anodical photocurrent across a $\mathrm{MoS}_{2}$ interface in contact with an acetonitril electrolyte $\left(0.3 \% \mathrm{H}_{2} \mathrm{O} ; 0.1 \mathrm{M} \mathrm{LiClO}_{4}\right)$; Reference electrode $\mathrm{Ag} / \mathrm{AgClO}_{4}$.

lyte. The anodical $\mathrm{MoS}_{2}$ photocurrents obtained with this electrolyte are very low and apparently due to traces of water. They can be made to rise to values observed with an aqueous electrolyte simply through addition of water. The same increase of the anodical photocurrent is also observed, if, instead of water, a reducing agent such as hydroquinone is added to the acetonitrile electrolyte. On illuminated
$\mathrm{MoS}_{2}$ surfaces water is, consequently, behaving photoelectrochemically like a simple reducing agent. In order to avoid that an increase of anodical photocurrents may be simulated by traces of water in the Ruthenium-complex, it was vacuum dried for several days at $50{ }^{\circ} \mathrm{C}$ and, in addition, $0.3 \%$ of water were allowed to remain in the acetonitril electrolyte to which the complex was to be added. The marked increase of anodical photocurrents (Fig. 7) to current densities of the order of $10^{-4}$ $\mathrm{A} / \mathrm{cm}^{2}$ proves the photoelectrochemical oxidation of $\mathrm{Ru}(\text { bipy })_{3}{ }^{2+}$ on $\mathrm{MoS}_{2}$ surfaces. The catalytic nature of the Ruthenium complex (Fig. $7 \mathrm{~b}$ ) in contrast to its sensitizing action (Fig. 6 a) has additionally been tested by measuring the spectral dependence of the generated photocurrent. Fig. 8 shows that in the absorption range of $\mathrm{Ru}(\text { bipy })_{3}{ }^{2+}$, where light is absorbed in a several millimeter thick layer of electrolyte, no photocurrent is observed. The generated photocurrents appear at longer wavelength and coincide there with the $\mathrm{MoS}_{2}$ photocurrent spectrum. Light absorption in the $\mathrm{MoS}_{2}$ surface with a subsequent hole reaction according to Fig. $7 \mathrm{~b}$ is thus confirmed. The mechanism of Fig. 6 a, sensitization by $\mathrm{Ru}$ (bipy) ${ }_{3}{ }^{2+}$ has been searched for at $\mathrm{ZnO}$ crystal surfaces. A sensitization effect, coinciding with the absorption of the complex, was actually observed. As expected, under comparable conditions, current densities were as low as $10^{-7} \mathrm{~A} / \mathrm{cm}^{2}$, that is approximately three orders of magnitude smaller than the photocurrents obtained through redox catalysis on $\mathrm{MoS}_{2}$ (Figure $6 \mathrm{~b}$ ).

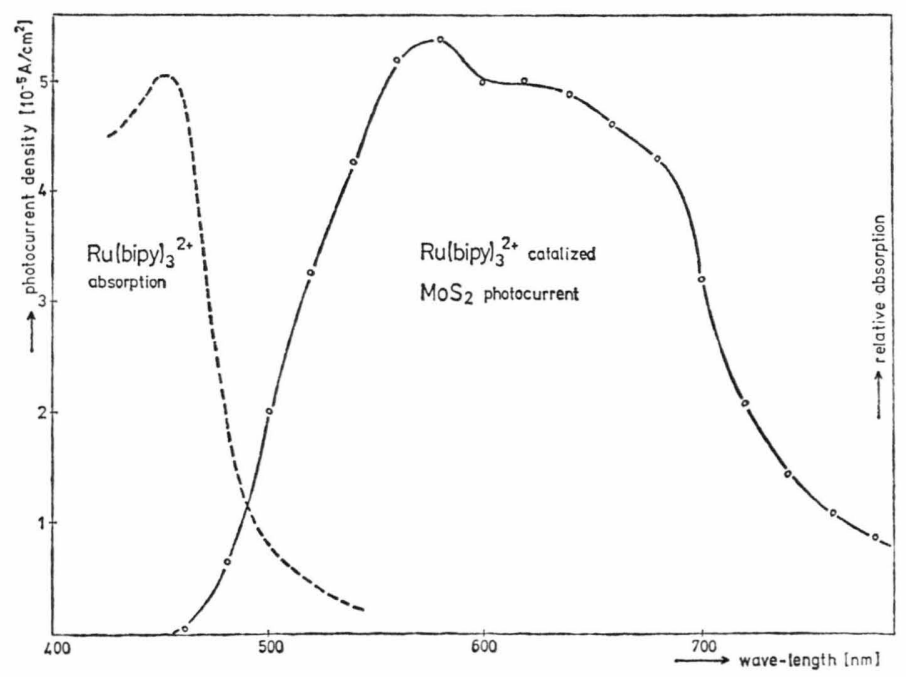

Fig. 8. Spectral dependence of Ru(bipy) ${ }_{3}{ }^{2+}$-photocurrents across a $\mathrm{MoS}_{2}$-acetonitrile $\left(0.3 \% \mathrm{H}_{2} \mathrm{O}\right.$; $0.1 \mathrm{M} \mathrm{LiClO}_{4}$ ) interface. Dotted curve: Absorption spectrum of $\mathrm{Ru}$ (bipy) ${ }_{3}{ }^{2+}$ which produces absorption loss in the blue spectral region. 


\section{Discussion}

In studies on energy conversion, the idea of looking for hole reactions from low-lying d-bands of transition metal dichalcogenides which represent non bonding orbitals had not been persued before. The discovery of a visible light induced photoelectrochemical mechanism leading to the oxidation of water at $\mathrm{MoS}_{2}$ electrode surfaces proves the theoretical concepts applied for the identification of a suitable substrate to be essentially correct.

Experiments have also confirmed the need for a transition metal compound in which the transition metal is able to undergo a valency change during the capture of a photogenerated hole. This implies that the hole has actually to be a missing electron belonging to a d-orbital of the metal and suggests the following mechanism: The original 4-valent state of Mo in the $\mathrm{MoS}_{2}$ surface is converted into a 5 valent one as a result of the capture of a hole. An additional bonding possibility is thus created: There will be some rearrangement of electron densities and atom positions and an $\mathrm{OH}^{-}$ion or water from the electrolyte may thus be chemically added to give a $\mathrm{Mo}(\mathrm{V})-\mathrm{OH}$ complex (Figure 9). This reaction possibility explains easily why an electron transfer from $\mathrm{OH}^{-}$or $\mathrm{H}_{2} \mathrm{O}$ can be initiated in such an energetically favourable way. This newly generated electronic $\mathrm{Mo}_{\mathrm{o}}(\mathrm{V})-\mathrm{OH}$ state will be situated somewhat above the Mo $-\mathrm{d}_{z^{2}}$ valence band of the $\mathrm{MoS}_{2}$ crystal (inserted energy scheme in Fig. 9) and will serve as a trap for a hole. If there is a missing electron in the lower Mo $4 \mathrm{~d}$ band, the $\mathrm{Mo}(\mathrm{V})-\mathrm{OH}$ state will be oxidized and the electron transfer from $\mathrm{OH}^{-}$into the $\mathrm{MoS}_{2}$ crystal thus completed. The proton will be dissociated and an instable oxide complex will be left which could release the oxygen either for a recombination with another oxygen surface-atom to an oxygen molecule or for a bond formation with surface-sulfur which could be stepwise oxidized to sulfate. Schematically, the reaction sequence can be described as follows:

$$
\begin{aligned}
& \mathrm{Mo}(\mathrm{IV})+\mathrm{p}^{+} \rightleftarrows \mathrm{Mo}(\mathrm{V})^{+} \\
& \mathrm{Mo}(\mathrm{V})^{+}+\mathrm{OH}^{-} \rightleftarrows \mathrm{Mo}(\mathrm{V})-\mathrm{OH} \\
& \begin{array}{l}
\mathrm{Mo}(\mathrm{V})-\mathrm{OH} \\
\mathrm{Mo}(\mathrm{IV})-\mathrm{S}
\end{array}+p^{+} \rightarrow \begin{array}{l}
\mathrm{Mo}(\mathrm{V}) \\
\mathrm{Mo}(\mathrm{IV})-\mathrm{S}^{\mathrm{O}+\mathrm{H}^{+}}
\end{array} \\
& \mathrm{Mo}(\mathrm{V})-\mathrm{OH} \quad \mathrm{Mo}(\mathrm{IV}) \quad \mathrm{O} \\
& \mathrm{Mo}(\mathrm{V})-\mathrm{OH} \stackrel{+2 p^{+}}{\mathrm{Mo}(\mathrm{IV})} \underset{\mathrm{O}}{+\left.\right|^{1}}+2 \mathrm{H}^{+}
\end{aligned}
$$

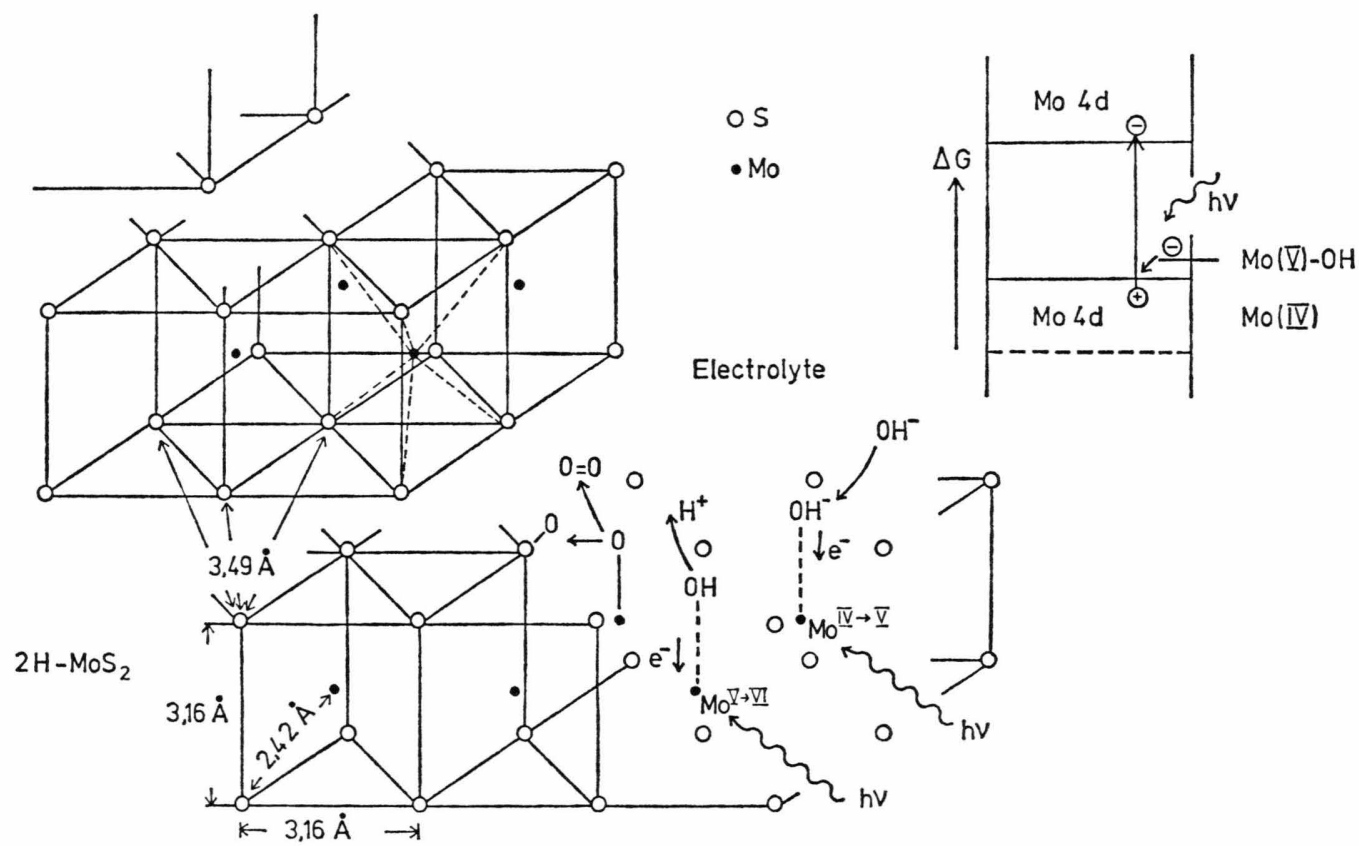

Fig. 9. Crystal structure of layer-type chalcogenide $\left(2 \mathrm{H}-\mathrm{MoS}_{2}\right)$ with schematical representation of $\mathrm{OH}^{-}$addition to oxidized Mo(IV), electron transfer, a subsequent oxidation step and oxygen liberation. Inserted is an energy diagram showing the position of $\mathrm{Mo}(\mathrm{V})-\mathrm{OH}$ transition state which is further oxidized. 
The critical step leading to the photoreaction with water is consequently a valency change of the transition metal which is creating a bonding possibility for $\mathrm{OH}^{-}$or $\mathrm{H}_{2} \mathrm{O}$. It is consequently not so much the relative position of electronic levels which is controlling the reaction, as the chemical bonding energy activated through the capture of a photogenerated hole. This explains why, with $\mathrm{MoSe}_{2}$, photons of as low as $1.5 \mathrm{eV}$ corresponding to infrared light up to $900 \mathrm{~nm}$ are able to induce a photoelectrochemical reaction with water.

There are circumstances which are suggesting that nature, in the oxygen evolution center of photosynthesis is, in principle, using the same chemical clue to accomplish an oxidation of water with visible or infrared light. A basic condition, the existence of a close neighbourhood of (two) transition metal ions which can change their oxidation state by two as proposed for the photosynthetic $\mathrm{Mn}^{++}$-centers by M. Calvin ${ }^{15}$ has been used as one criterion to identify $\mathrm{MoS}_{2}$ as a possible substrate for the photooxidation of water. The positive results obtained with layer type crystals of group VI-metals indicate that Calvin's concept is, in principle, correct. However, according to our considerations, it would have to be supplemented in one detail: Calvin suggests a hole mediated successive oxidation of a $\mathrm{Mn}^{++} \ldots \mathrm{Mn}^{++}$pair to $\mathrm{Mn}^{4+} \ldots \mathrm{Mn}^{4+}$ before two 2 electron transfer steps from water would lead to oxygen evolution. Though theoretically not impossible, 2-electron steps are kinetically too limiting nor has such a case yet been convincingly described in the scientific literature. In order to overcome the energetical barrier for a one step-electrontransfer from $\mathrm{OH}^{-}$or water, it is necessary to assume - as shown in the investigation - that the first oxidation state of the transition metal centers, that is $\mathrm{Mn}^{3+}$ in the photosynthetic apparatus, is forming a chemical bond with $\mathrm{OH}^{-}$. A pair of such $\mathrm{Mn}^{3+}-\mathrm{OH}$ could easily be further oxidized to yield free molecular oxygen. Other qualitative similarities of the photooxidation of water in photosynthesis and on $\mathrm{MoS}_{2}$ surfaces concerning the

1 A. Fujishima and K. Honda, Nature 238, 37 [1972], and Bull. Chem. Soc. Japan 44, 1148 [1971].

2 J. Keeny, D. H. Weinstein, and G. M. Haas, Nature 253, 719 [1975].

3 M. S. Wrighton, D. S. Ginley, P. T. Wolczanski, A. B. Ellis, D. L. Morse, and A. Liwz, Proc. Nat. Acad. Sci USA 72, 1518 [1975]. basic character of the mechanism include the effectiveness of visible as well as infrared light and the possibility of replacing water for typical electron transfer agents in the oxidation reaction. If these considerations are correct, it should be promising to work on a new type of synthetical substrates for lightinduced oxidation of water. As in $\mathrm{MoS}_{2}$ and $\mathrm{MoSe}_{2}$ photogenerated holes have to be made available at sufficiently positive energy levels for an oxidation of a transition metal of group VI to VIII a (Figure 2). An interesting choice would be a suitable semiconductor with a IV-valent metal constituent into which VI-valent transition metal ions would be incorporated. The oxidation of $\mathrm{OH}^{-}$ should then proceed stepwise, involving a hydroxide bond in a higher valency state of the transition metal. The most difficult problem will be the chemical stabilization of the interface against attack from atomic oxygen which is formed as an intermediate product loosely bound to the transition metal. With $\mathrm{MoS}_{2}$ and $\mathrm{MoSe}_{2}$ this stability is not given but can, for example, to some extent be improved with the help of a redox catalyst $\left[\mathrm{Ru}(\text { bipy })_{2}{ }^{3+}\right]$ which is channelling reactive holes into a better controlled chemical reaction with water.

The problem of an artificial visible light induced oxidation of water similar to that in photosynthesis is generally considered too difficult to encourage substantial research efforts. This investigation shows that some progress can be accomplished already with relatively modest theoretical and experimental efforts.

\section{Acknowledgement}

The work became possible through a grant from the Deutsche Forschungsgemeinschaft. Its support by Professon Gerischer and his constructive criticism are gratefully acknowledged. The author thanks Mrs. C. Lober and Mr. J. C. Bennett for valuable technical assistance, Dr. J. Holzwarth for the $\mathrm{Ru}$ (bipy) ${ }_{3}{ }^{2+}$-complex and Mrs. M. Lübke for performing the $\mathrm{Ru}$ (bipy) ${ }_{3}{ }^{2+}$ sensitization experiment with $\mathrm{ZnO}$.

4 S. N. Frank and A. J. Bard, J. Amer. Chem. Soc. 97, 7427 [1975].

5 K. L. Hardee and A. J. Bard, J. Electrochem. Soc. 122, 739 [1975].

6 A. J. Nozik, Nature 257, 383 [1975].

7 H. Yoneyama, H. Sakamoto, and H. Tamura, Electrochim. Acta 20,341 [1975]. 
8 A. Fujishima, K. Kohayakawa, and K. Honda, Bull. Chem. Soc. Japan 48, 1041 [1975], and J. Electrochem. Soc. 122, 1437 [1975].

9 W. Gissler, P. L. Lensi, and S. Pizzini, J. Appl. Electrochem. 6, 9 [1976].

10 M. S. Wrighton, D. L. Morse, A. B. Ellis, D. S. Ginley, and H. B. Abrahamson, J. Amer. Chem. Soc. 98, 44 [1976].

11 M. S. Wrighton, A. B. Ellis, P. T. Wolczanski, D. L. Morse, H. B. Abrahamson, and D. S. Ginley, J. Amer. Chem. Soc. 98, 2774 [1976].

12 J. G. Mavroides, J. A. Kafalas, and D. F. Koselar, Appl. Phys. Lett. 28, 241 [1976].

13 A. B. Ellis, S. W. Kaiser, and M. S. Wrighton, J. Phys. Chem. 80, 1325 [1976].

14 Yoshihiro Nakato, Koichi Abe, and Hiroshi Tsubomura, Ber. Bunsenges. phys. Chem. 80, 1002 [1976].

15 M. Calvin, Science 184, 375-381 [1974].

16 C. Creutz and N. Sutin, Proc. Nat. Acad. Sci. (USA) 72, $2858-2862$ [1975].

17 H. Tributsch, Bioenergetics 2, 249 [1971]; Photochem. Photobiol. 16, 261 [1972].

18 J. A. Wilson and A. D. Yoffe, Adv. Phys. 18, 193 [1969].

19 Paul Ramdohr, Die Erzmineralien und ihre Verwachsun. gen, Akademieverlag, Berlin 1960.
20 A. F. Wells, Structural Inorganic Chemistry, Clarendon Press, Oxford 1975.

21 L. F. Mattheiss, Phys. Rev. 8 D, 3719 [1973].

22 R. T. Shuey, Semiconducting ore minerals Elsevier Scientific Publishing Company, Amsterdam, Oxford, New York 1975.

23 P. M. Williams and F. R. Shepherd, J. Phys. C 6, L 36L 40 [1973].

24 R. V. Kasowski, Phys. Rev. Letters 30, 1175 [1973].

25 K. Wood and J. B. Pendry, Phys. Rev. Letters 31, 1400 [1973].

26 R. S. Title and M. W. Shafer, Phys. Rev. Letters 28, $808-810$ [1972].

$27 \mathrm{H}$. Tributsch and J. C. Bennett, J. Electroanal. Chem. [1977], in press.

28 A. Albert, Angew. Chem. Int. Ed. Engl. 6, 919 [1967].

29 G. Navon and N. Sutin, Inorganic Chemistry 13, 2159 [1974].

30 C. Creutz and N. Sutin, Proc. Nat. Acad. Sci. USA 72, 2858 [1975].

$31 \mathrm{H}$. Tributsch, to be published.

32 J. C. Bennett, B. Pettinger and H. Tributsch, to be published. 